distorsion est de quelques pour cent seulement pour $8542 \mathrm{CaII}$, avec $L>\mathrm{x} \cdot 000 \mathrm{~km}$ et une amplitude inférieure à $2 \mathrm{~km} / \mathrm{s}$ dans la zone de formation de la raie.

Nous pouvons écrire:

$$
d(t) \simeq \frac{\lambda}{c} a \rho\left(z_{0}\right)^{-\frac{1}{2}} g(L) \cos 2 \pi\left[\frac{t}{P}-\frac{z_{\phi}(L)}{L}\right]
$$

Dans cette expression, nous choisissons l'altitude $z_{0}$ de manière à ajuster la fonction $g(L)$ à l'unité pour $L$ infini. Cette altitude se place alors au voisinnage du minimum de température pour $85^{\mathrm{I}} 4 \mathrm{FeI}$, et environ $900 \mathrm{~km}$ au-dessus pour $8542 \mathrm{CaII}$.

La quantité $z_{\phi}(L)$ est une 'altitude de formation de la phase' et peut être reliée aux calculs de corrélations croisées portant sur les deux raies.

La fonction $g(L)$ décrit l'atténuation apparente des vitesses par intégration sur la ligne de visée; $g^{2}(L)$ décroît lorsque $L$ décroit, et atteint la valeur I $/ 2$ vers $L=700 \mathrm{~km}$ pour $85 \mathrm{I} 4 \mathrm{Fe}$, et $L=2300 \mathrm{~km}$ pour 8542 CaIr. Malheureusement, chaque période $P$ est associée en fait à un domaine de valeurs de $L$ (ondes obliques). La répartition spectrale observée est donc égale, pour chaque période, au produit de la répartition réelle en $z_{0}$ par la valeur que prend $g^{2}$ pour un $L$ moyen inconnu. Nous pouvons néanmoins faire deux remarques qualitatives:

I. Considérons une longue période, $280 \mathrm{~s}$ par exemple, voisine de la période critique. Les longueurs d'onde correspondantes sont très grandes, et $g(L)$ toujours peu différent de $\mathrm{I}$. Or, l'amplitude observée croît environ d'un facteur 2 d'une raie à l'autre, alors que le coefficient $\rho\left(z_{0}\right)^{-\frac{1}{2}}$ croît d'un facteur 30 . On peut penser que la période critique moyenne pour la basse chromosphère se situe légèrement au-dessous de $280 \mathrm{~s}$, et que les ondes observées sont principalement stationnaires et amorties.

2. Pour une courte période, I50 s par exemple, la valeur moyenne de $L$ est probablement inférieure à $2000 \mathrm{~km}$, malgré la proximité de la période critique. Dans le cas de $8542 \mathrm{CaII}$, le rapport entre puissance spectrale réelle et puissance spectrale observée apparaît supérieur à 2 .

Il est donc vraisemblable que les ondes de haute fréquence transportent dans la chromosphère une proportion importante d'énergie mécanique.

\title{
BIBLIOGRAPHIE
}

I. Evans, J., Michard, R., Servajean, R. Ann. Astrophys., 26, 368, 1963.

\section{THE HEATING OF THE SOLAR CORONA BY PHOTOSPHERIC WAVES}

\author{
M. Kuperus \\ (Utrecht Observatory)
}

In the turbulent boundary of the convection zone a flux of pressure waves is generated by the compressible turbulence. It can be shown that nearly the whole flux is radiated from a thin layer of about $100 \mathrm{~km}$ thickness at a depth of about $400 \mathrm{~km}$. This flux is of the order of $10^{7}$ ergs $\mathrm{cm}^{-2} \mathrm{sec}^{-1}$ and depends largely on the convective velocities.

In the photosphere and low chromosphere mechanical energy is mainly transported by two kinds of waves; internal gravity waves and acoustic waves. It is found that below a critical 
frequency the waves are essentially internal gravity waves modified by the compressibility and above another critical frequency they are acoustic waves influenced by the stratification of the atmosphere. Close to the critical frequencies the energy transfer is predominantly vertical. In between the two critical frequencies travelling waves are excluded. Both types of waves show an exponential increase in amplitude during their journey upwards caused by the decrease in density. Because of this increase in amplitude the waves become more and more non-linear until already after 4-5 scale heights they have been transformed into shock waves.

The temperature rises due to the efficient energy dissipation in the shock waves. The dissipated energy is mainly radiated but as soon as the density decreases below a certain value the dissipation exceeds the radiation and the temperature has to increase steeply so that backward heat conduction can balance the energy supply.

It is possible to compute the coronal temperature and the temperature distribution in the transition region between the chromosphere and the corona, if one drastically simplifies the atmosphere, first by neglecting the inhomogeneous structure and secondly by the the assumption that the wave propagation could be treated essentially one-dimensional.

The physical conditions in the transition region are governed by the following four differential equations:

$$
\begin{aligned}
& \frac{\mathrm{d} F_{\mathrm{m}}}{\mathrm{d} z}=-\kappa F_{\mathrm{m}}-\frac{F_{\mathrm{m}} \frac{\mathrm{d} T}{T} \frac{\mathrm{d} z}{\mathrm{~d}},}{\frac{\mathrm{d} F_{\mathrm{c}}}{\mathrm{d} z}=E_{\mathrm{rad}}-E_{\mathrm{diss}},} \\
& \frac{\mathrm{d} T}{\mathrm{~d} z}=\text { const } \times T^{-5 / 2} F_{\mathrm{c}}, \\
& \frac{\mathrm{d} n}{\mathrm{~d} z}=-n\left(\frac{\mathrm{I}}{H}+\frac{\mathrm{I}}{T} \frac{\mathrm{d} T}{\mathrm{~d} z}\right),
\end{aligned}
$$

where $E_{\mathrm{rad}}=$ const $\times n^{2}, E_{\mathrm{diss}}=\kappa \times F_{\mathrm{m}}$ and $\kappa=$ const $\times \frac{M^{2}-\mathrm{I}}{c \times P \times M^{3}}$

is the absorption coefficient, $H$ is the scale height, $P$ the period of the waves, $c$ the velocity of sound, $M$ the Mach number of the shock waves, $F_{\mathrm{m}}$ and $F_{\mathrm{c}}$ the mechanical and conductive energy flux. Equation ( 1 ) results from an approximate expression of the mechanical energy flux taking into account the reflection of shock waves in the solar atmosphere.

$$
F_{\mathrm{m}}(z)=F_{\mathrm{m}}(0) \times \exp \left\{-\int_{0}^{z} \kappa \mathrm{d} z \times \frac{T(0)}{T(z)}\right\}
$$

The integration of the equations is started in the corona, where $\frac{\mathrm{d} T}{\mathrm{~d} z}=F_{\mathrm{o}}=0$, for various coronal temperatures and densities going downward in the atmosphere. They were stopped when the temperature becomes below $50000^{\circ} \mathrm{K}$. The result of a number of integrations is shown in Fig. I.

The particle density at the $50000^{\circ} \mathrm{K}$ level is plotted against the mechanical energy flux that should be available at this level in order to maintain a solar corona characterized by the density and temperature, represented by the solid lines in the diagram. For a number of models of the high chromosphere relating the density, the temperature and the height above the photosphere equation (5) yields the mechanical energy flux at the $50000^{\circ} \mathrm{K}$ level. The open circle (A) serves as a mean for these models. We find a temperature of about $T=700000^{\circ} \mathrm{K}$ together with a 


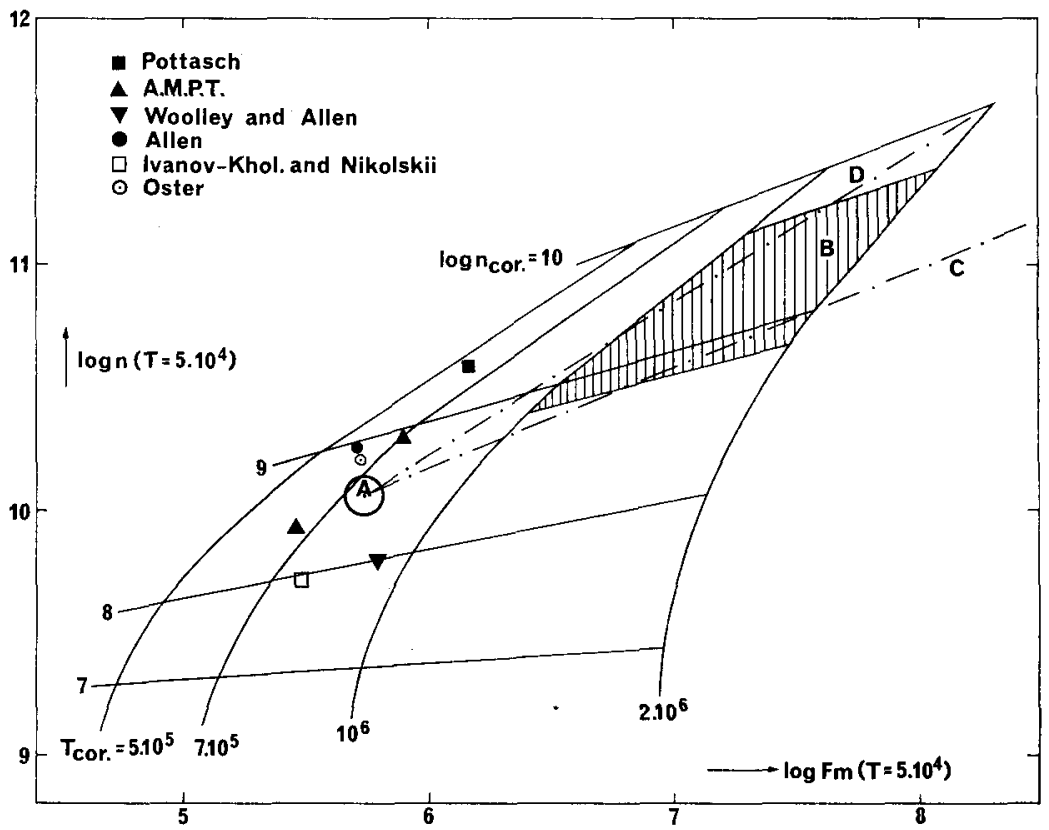

FIG. I

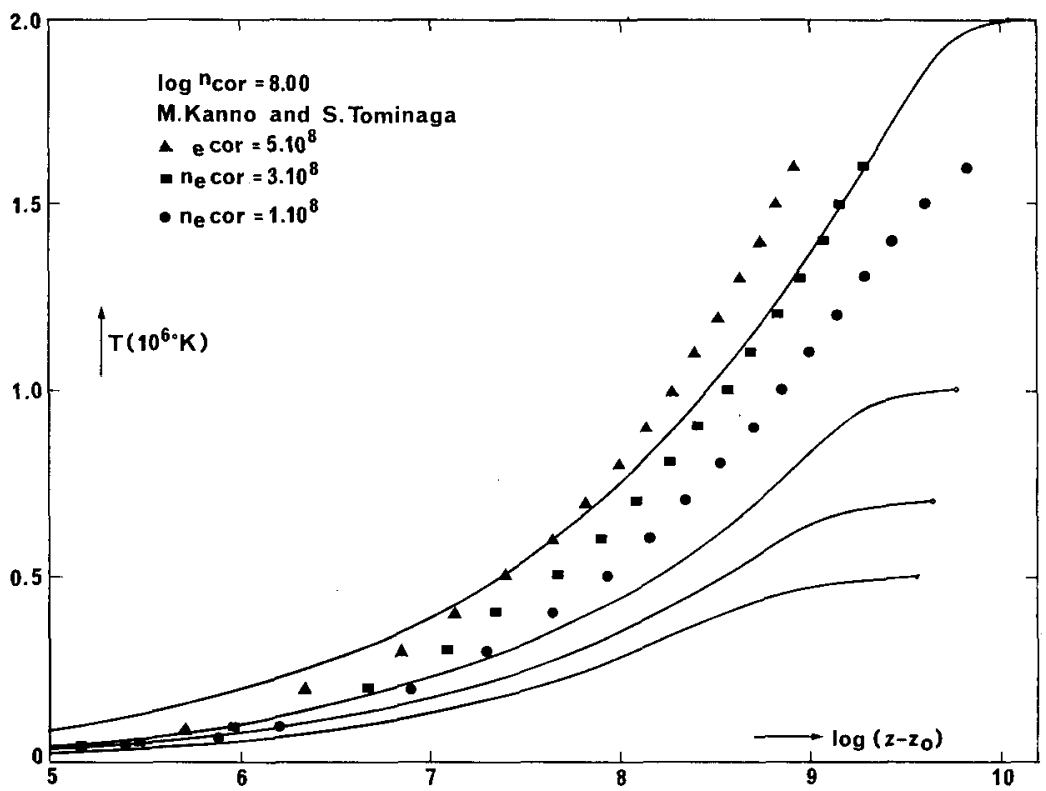

FIG. 2 
particle density $n_{\mathrm{cor}}=4 \times 10^{8} \mathrm{~cm}^{-3}$ for the undisturbed corona, which is in quite good agreement with the observations. Nevertheless the uncertainties are very large because of the poor knowledge of $F(0)$ and $\kappa$.

It is possible that monopole and dipole emission play an important part in the energy generation, so that the computed coronal values should be seen as lower limits.

All integrations point towards a very steep temperature increase between the chromosphere and the corona so that the transition region is an extremely thin layer of some hundred $\mathrm{km}$.

Fig. 2 shows the temperature versus $\log \left(z-z_{0}\right)$ for $\log n=9 \cdot 00, z_{0}$ being the height where the integrations were stopped. Recently M. Kanno and S. Tominaga (I) constructed a model of the transition region based on observational data. Their three models are compared in Fig. 2 with our computations and a fairly good agreement is found concerning the temperature gradients.

For the corona above a centre of activity one normally finds a density increase up to a factor of 10. The temperature is assumed to be somewhat higher $\left(10^{6}-2 \cdot 10^{6} \mathrm{~K}\right)$. Those active coronal values are represented in the diagram by the hatched curved quadrangle $B$. The two broken lines $\mathrm{C}$ and $\mathrm{D}$ indicate how the density at the $50000^{\circ} \mathrm{K}$ level increases as the mechanical energy flux increases. The line $\mathrm{C}$ gives the relation for very high Mach numbers $M \gg \mathrm{I}$ and the line $\mathrm{D}$ for $M$ close to unity.

It seems likely that photospheric turbulent magnetic fields in activity regions will enhance the flux of mechanical energy by a factor of the order of 10 (Kulsrud (z)). However other wave modifications introduced by the important magnetic fields will play a part so that a new diagram should be made for these kinds of waves.

Nevertheless it looks as if the permanent long lasting coronal condensations could be built up and maintained by an intensification of the same processes, which are responsible for the existence of the quiet corona.

\section{REFERENCES}

I. Kanno, M., Tominaga, S. Publ. astr. Soc. Fapan. To be published.

2. Kulsrud, R. M. Astrophys. F., 121, 461, 1955.

\section{DISCUSSION}

$Y$. Uchida. You mentioned the effect of a magnetic field on the generation of kinetic energy flux. I think the effect of it on the propagation could also make the temperature of the corona higher. If we consider a decreasing magnetic field with height, it suppresses the heat liberation in the lower layers where the radiative loss is efficient, and the region of dissipation is shifted into a little higher layer. This makes the heat conduction larger, which so determines the temperature gradient in wider range of height.

C. de fager. The results of Osterbrock's computation show that coronal heating by hydromagnetic waves in a facular region does not occur since the waves are refracted backward. Since, on the other hand, coronal active regions seem hotter than the quiet corona, the heating of coronal active regions must occur through dissipation of waves of the acoustic type.

$M$. Kuperus. In a magnetic field most of the mechanical energy is transported by the fastmode magnetohydrodynamical waves. Nevertheless the waves are already transformed into fast-mode shocks at very low levels, so that the simple refraction laws for small amplitude waves may hardly be used. Moreover waves which travel into the direction of the field can be treated as normal gasdynamic shocks. In this case the magnetic field has no influence on the mechanical energy flux. 
F. Kahn. You have calculated what occurs when a wave travels straight upwards into the corona. Can anything be said about waves which travel at an angle to the vertical?

$M$. Kuperus. For the propagation of shock waves in an arbitrary direction it is difficult to predict the loss of energy flux by refraction and reflexion, because we cannot apply geometrical acoustics to the propagation of non-linear waves. However, one can see qualitatively that nonlinear waves are in a favourite position as compared with linear waves with respect to the conservation of flux.

C. de fager. Neven and I, a few years ago (Liège colloquium on ultra-violet emission of celestial bodies) computed theoretical stellar coronas and their emission. The computations should now be remade in view of the advance theory has since made, and by using the new information on stellar photospheric turbulence.

A. Deutsch. For the spectroscopically observed envelopes of red giants, which may also be regarded as a kind of stellar coronae, Weyman has shown that electron conduction is no longer the dominant process of energy transport. It would be desirable, if Weyman's models for these stellar coronas could be elaborated by the methods Dr Kuperus has described here. 